\title{
Perfil de líderes en oficinas de transferencia tecnológica en instituciones de educación superior mexicanas
}

\author{
Profile of leaders in technology transfer offices in mexican \\ higher education institutions \\ DOI: $10.22458 /$ rna.v12i 1.3460
}

\begin{abstract}
Dr. Alfredo Pérez Paredes ${ }^{1}$
Dra. Diana Barrón Villaverde ${ }^{2}$

Dr. José Aurelio Cruz de los Ángeles ${ }^{1}$

${ }^{\mathbf{1} B e n e m e ́ r i t a ~ U n i v e r s i d a d ~ A u t o ́ n o m a ~ d e ~ P u e b l a, ~ F a c u l t a d ~ d e ~ A d m i n i s t r a c i o ́ n, ~ P u e b l a, ~ M e ́ x i c o ; ~ a l f r e d o . p e r e z @ c o r r e o . b u a p . m x, ~}$ https://orcid.org/Oooo-0001-8766-5766; joseaurelio.cruz@correo.buap.mx, https://orcid.org/oooo-0002-8682-366X

${ }^{\mathbf{2}}$ Universidad Popular Autónoma del Estado de Puebla, Decanato de Ingeniería, Posgrado de Planeación Estratégica y Dirección de Tecnología, Puebla, México, diana.barron@upaep.mx, https://orcid.org/0000-0003-2329-362X
\end{abstract}

\section{RESUMEN}

A pesar de existir investigaciones sobre transferencia tecnológica (TT), son contados los que analizan las habilidades gerenciales o técnicas de los líderes y nulos en latitudes de países emergentes. Es por ello que, particularmente, se estudia el caso de México. Este artículo analiza cuáles habilidades son las que predominan en el perfil del agente de oficinas de transferencia tecnológica universitarias en México. Por medio de un análisis estadístico multifactorial, se identificaron ocho componentes que contienen, en un 79,4\%, ocho constructos. Como resultado, se muestra la identificación del perfil de agente de transferencia.

\section{RÉSUMÉ}

Bien qu'il y ait de la recherche liée au transfert technologique (TT), il y a très peu d'articles sur les compétences managériales ou techniques de dirigeants et aucun dans les pays émergents. C'est pourquoi, cet article analyse le cas du Mexique et se concentre particulièrement sur les compétences prédominantes dans le profil des agents de bureaux de transfert de technologie dans les universités mexicaines. Grâce à une analyse statistique multifactorielle, huit composantes ont été identifiées, contenant huit concepts à $79,4 \%$. Par conséquent, est montrée l'identification du profil d'agent de transfert.

\section{RESUMO}

Embora existam investigações sobre transferência de tecnologia (TT), são poucas as que analisam as competências gerenciais ou técnicas de líderes e nenhum em latitudes de países emergentes. É por isso que, em particular, se estuda o caso do México. Este artigo analisa quais competências são as que predominam no perfil do agente de escritórios universitários de transferência tecnológica no México. Por meio de uma análise estatística multifatorial, foram identificados oito componentes que contêm, em $79,4 \%$, oito construtos. Como resultado, é amostrada a identificação do perfil do agente de transferência.
PALABRAS CLAVE:

TRANSFERENCIA

TECNOLÓGICA, OFICINAS,

AGENTE, INSTITUTOS DE

EDUCACIÓN SUPERIOR

(IES), GESTIÓN

DEL CAPITAL HUMANO.
KEYWORDS:

TECHNOLOGY TRANSFER

OFFICES, AGENTS,

HIGHER EDUCATION

INSTITUTES, HUMAN

CAPITAL MANAGEMENT.
MOTS CLÉS:

TRANSFERT DE

TECHNOLOGIE, BUREAUX,

AGENT, INSTITUTS

D'ENSEIGNEMENT

SUPÉRIEUR (IES), GESTION

DU CAPITAL HUMAIN.
PALAVRAS-CHAVE:

TRANSFERÊNCIA

TECNOLÓGICA,

ESCRITÓRIOS, AGENTE,

INSTITUTOS DE ENSINO

SUPERIOR (IES), GESTÃO

DE CAPITAL HUMANO. 


\section{INTRODUCCIÓN}

La transferencia tecnológica (TT) es el proceso en el que se facilitan habilidades, conocimiento, tecnologías, métodos de fabricación, muestras de fabricación e instalaciones entre los gobiernos o las universidades (Cursi, 2021). De acuerdo con Farda et al. (2014), la TT tiene un efecto indirecto como facilitador y acumulador de las capacidades de absorción de la industria, debido a que la nueva economía basada en conocimiento promueve el flujo de ideas. Este proceso tiene como principal cometido el valorizar el conocimiento para que las empresas lo conviertan en productos o servicios comercializables (Siegel et al., 2003). Dentro de los diferentes modelos de transferencia tecnológica existentes, en esta investigación se ahondará en la relación de TT entre la universidad y la industria.

La comunidad universitaria ha demostrado que el emprendimiento cubre gran parte de la realización, aplicación y ejecución de ideas. Incluso las redes universitarias han logrado pasar de los modelos teóricos a los ejemplos tangibles de los negocios que resuelven y superan la creencia de que solamente son las organizaciones dedicadas a los servicios las únicas promotoras de emprendimientos (García, 2019).

Recientemente, las universidades han adquirido diversas funciones adicionales a la de formar estudiantes y realizar investigación, entre ellas se encuentran: identificar nuevas fuentes de fondeo para investigaciones o instalaciones en la universidad (Vutsova y Ignatova, 2014); comercializar el conocimiento académico (O’Kane, et al., 2015) a través de servicios de consultoría, licenciamientos o creación de empresas spin outs, lo que se ha denominado su tercera misión (Vorley T. and Nelles J., 2009; Rothaermel et al., 2007).

La relación entre el sector industrial y la academia ha sido concebida como fundamental para lograr tanto mayor desarrollo económico (Casas, 1999; OCDE, 2009; Riviezzo y Napolitano, 2010) como tecnológico de las naciones (Griliches, 1992; UN, 2015; Pagar, K, et al., 2014). Por ello, la transferencia tecnológica (TT) se ha concebido crecientemente como un pivote del desarrollo a nivel macroeconómico y ha sido a través de las Oficinas de Transferencia de Tecnología (OTT) que se ha fortalecido e impulsado esta relación, que estimulan la diseminación y comercialización de la investigación; de esta forma es posible trasladar las investigaciones a la práctica; y facilitar el fortalecimiento con la interrelación con la industria y sociedad como agentes que forman parte del sistema de innovación (Algieri et al., 2013).

Dado que ya existe documentación relacionada con las funciones de las OTT, su desempeño, evaluación de las mismas y modelos para su gestión, este artículo indaga sobre un actor fundamental de la OTT: el agente de transferencia tecnológica. Entre las preguntas principales que se busca responder: ¿existe un perfil determinado para la gerencia en la transferencia tecnológica en México? ¿cuáles son las características que predominan? y ¿existe alguna jerarquización de las capacidades?

Es por ello por lo que, en esta investigación, se identificaron las habilidades técnicas y gerenciales preponderantes en el perfil del agente de TT de las OTT pertenecientes a universidades mexicanas. Este estudio es particularmente útil dado que a la fecha no existen suficientes estudios similares en México, por lo que se justifica generar información que ayude a las actividades de formación, reclutamiento, capacitación y evaluación de los agentes tecnológicos.

El estudio incluyó una revisión sobre literatura concerniente a las habilidades tanto gerenciales como técnicas de los agentes de transferencia tecnológica. De igual forma, se analizó el perfil de participación de los agentes de transferencia de las oficinas universitarias de transferencia tecnológica en México. Para tal fin, se creó un instrumento explícitamente para identificar y jerarquizar las principales características de dichos agentes, el cual se aplicó a través de encuestas en línea a las OTT de universidades dentro del territorio nacional. Finalmente, se analizaron los resultados obtenidos de las entrevistas con análisis multifactorial para identificar si son más preponderantes las habilidades técnicas o gerenciales en el perfil del agente.

\section{Revisión de la literatura.}

Entre los investigadores de los programas de transferencia tecnológica de las universidades y las industrias (Berazael et al., 2015; Villani et al., 2015), se ha analizado la necesidad de las OTT como vehículos para trasladar el conocimiento de las instituciones generadoras a las usuarias de conocimiento (Suvinen et al., 2010); estrategias para su desarrollo (Franks \& Bory, 2017); así como, su efectividad en el apoyo a la transferencia (Harman, 2010; Olaya, Berbegal-Mirabent y Germán, 2014; Ustundag et al., 2011); y la intervención de estos intermediarios en sectores específicos como el de baja tecnología (Spithoven \& Knockaert, 2012), en la industria textil brasileña 
(Junior, et al., 2014). No obstante, son escasos los estudios enfocados a estudiar particularmente las habilidades gerenciales o personales para transferir tecnología. Se han realizado estudios sobre las actitudes y habilidades para los emprendedores de base tecnológica (Marion et al., 2012; Hrehová y Jencová, 2014; González, 2015), indicando la mayoría que son indispensables: la experiencia, formación académica, conocimientos y habilidades de gestión tecnológica y vinculación con la industria. En particular sobre el agente de transferencia tecnológica se encuentra el estudio de Harman y Stone (2006) que muestra cifras a nivel nacional que describen e identifican la ocupación, responsabilidades y características de los oficiales. Solleiro (2010) hizo una propuesta de capacidades técnicas y conocimientos que deben tener el personal de las OTT que promueve la transferencia de tecnología. Por su parte Mon et al. (2012), estudian las habilidades requeridas para los agentes; sin embargo, su estudio es general y éstas no se encuentran categorizadas al interior. Es por ello que este artículo brinda la pauta para comprender las habilidades necesarias para un agente de transferencia tecnológica, particularmente en la geografía mexicana. Específicamente para este estudio, se consideraron las siguientes variables, con los respectivos autores.

\section{Tabla 1}

Habilidades técnicas y gerenciales del estado del arte

\begin{tabular}{|c|c|c|c|c|c|}
\hline \multicolumn{3}{|c|}{ Habilidades técnicas } & \multicolumn{3}{|c|}{ Habilidades gerenciales } \\
\hline Conocimientos: & $\begin{array}{l}\text { de negocios } \\
\text { tecnológicos y } \\
\text { comunicación - } \\
\text { segundo idioma, } \\
\text { inglés- } \\
\text { Gestión } \\
\text { tecnológica, } \\
\text { propiedad } \\
\text { intelectual, } \\
\text { inteligencia } \\
\text { tecnológica y } \\
\text { de mercado, } \\
\text { contratos }\end{array}$ & (Leiponen, 2005) & $\begin{array}{l}\text { Clima laboral } \\
\text { Negociación, } \\
\text { solución de } \\
\text { conflictos } \\
\text { Elaboración e } \\
\text { interpretación de } \\
\text { textos complejos } \\
\text { Toma de decisiones }\end{array}$ & $\begin{array}{l}\text { Establecer un } \\
\text { ambiente de } \\
\text { cordialidad en el } \\
\text { ámbito laboral. } \\
\text { Organización de } \\
\text { grupos de trabajo } \\
\text { Adaptabilidad a } \\
\text { ambientes diversos } \\
\text { Fuerte orientación a } \\
\text { resultados }\end{array}$ & $\begin{array}{l}\text { (Lichtenthaler \& } \\
\text { Ernst, 2009) } \\
\text { Solleiro (2009) }\end{array}$ \\
\hline $\begin{array}{l}\text { Procedimientos } \\
\text { internos - } \\
\text { normatividad- }\end{array}$ & $\begin{array}{l}\text { libertad } \\
\text { suficiente en } \\
\text { mi IES para } \\
\text { TT de mi OTT }\end{array}$ & $\begin{array}{l}\text { (O'Kane, } \\
\text { Mangematin, } \\
\text { Geoghegan, \& } \\
\text { Fitzgerald, 2015) }\end{array}$ & $\begin{array}{l}\text { Emprendedurismo } \\
\text { corporativo }\end{array}$ & $\begin{array}{l}\text { Empresas creadas a } \\
\text { partir de desarrollos } \\
\text { tecnológicos en } \\
\text { universidades. }\end{array}$ & $\begin{array}{l}\text { (Corsi \& Prencipe, } \\
2016) \\
\text { (Drucker, 1985) }\end{array}$ \\
\hline $\begin{array}{l}\text { Propiedad } \\
\text { industrial }\end{array}$ & $\begin{array}{l}\text { Conocimiento } \\
\text { sobre } \\
\text { normatividades } \\
\text { de } \\
\text { patentamiento }\end{array}$ & $\begin{array}{l}\text { (ESTREICHER \& } \\
\text { YOST, 2016) } \\
\text { (Triiillo\& Everett, } \\
2010 \text { ) }\end{array}$ & Ética & $\begin{array}{l}\text { Códigos de conducta } \\
\text { ante patentamiento. }\end{array}$ & $\begin{array}{l}\text { (Sterckx, } 2011 \text { ) } \\
\text { (WOLFE, et al., } \\
2014)\end{array}$ \\
\hline $\begin{array}{l}\text { Planeación: } \\
\text { anticipación de } \\
\text { riesgos. }\end{array}$ & $\begin{array}{l}\text { Previsión } \\
\text { de fases } \\
\text { posteriores. }\end{array}$ & $\begin{array}{l}\text { (Makarov \& } \\
\text { Plantin, 2015) }\end{array}$ & Liderazgo & $\begin{array}{l}\text { Influir en otros tanto } \\
\text { horizontal como } \\
\text { verticalmente. }\end{array}$ & $\begin{array}{l}\text { (Sarin \& } \\
\text { McDermott, 2003) }\end{array}$ \\
\hline
\end{tabular}

Fuente: elaboración propia a partir de autores mencionados dentro de la tabla.

Dado que los líderes se encargan de tomar las decisiones dentro de sus respectivas organizaciones, son ellos mismos los que determinan el éxito en el logro de las metas propuestas. Por ello, se requiere que un líder tenga la posibilidad de tomar decisiones y que sea capaz de realizar una negociación, entre diversas habilidades que se consideran necesarias para identificar el camino idóneo para su organización. Precisamente en esto reside la relevancia de identificar y comprender dichas habilidades, dado que la carencia de comprensión al respecto ocasionaría una coordinación fallida y una inadecuada comunicación; falta de retroalimentación o planeación de desarrollo profesional; incentivos carentes de relevancia para los investigadores, entre otros inconvenientes (Marion et al., 2012; Hrehová y Jencová, 2014). 


\section{Contexto mexicano de las OT de instituciones de educación superior (IES).}

Existe un proceso débil de TT entre los sectores científico e industrial (Feria e Hidalgo, 2011). En gran medida, indican los autores, se debe al papel del espectador que han jugado las IES en el desarrollo económico e industrial del país al conformarse tan sólo con proveer capital humano a las empresas. El resultado de no involucrarse ha sido una amplia brecha entre la generación, transformación y aplicación de conocimiento.

De acuerdo con Beltrones et al. (2017), existe una mayor colaboración entre universidad e industria en actividades que requieren menor inversión y acuerdos de baja complejidad. La mitad (54.54\%) de las IES realiza actividades de investigación, desarrollo experimental o innovación no comisionados por empresas u organismos. El 36.17 \%de las IES encuestadas ofrecen servicios tecnológicos al mismo sector. Sólo 16.31 \% contaría con incubadoras de empresas.

La vinculación entre IES y el sector industrial tiene pocos efectos directos en competitividad e innovación empresarial. De acuerdo con Cabrero et al., (2011), estos mismos autores clasificaron los inhibidores de la actividad de colaboración entre IES e industria en tres categorías:

- Organización interna: asincronía entre calendarios de ambos sectores, desinterés de parte de la industria, falta de institucionalización de las IES en aspectos de normatividad como falta de manuales y procedimientos para gestionar proyectos de I+D o falta de normatividad para ejercer recursos externos a las IES.

- Comunicación: desconocimiento de la oferta de I+D de las IES.

- Disponibilidad de recursos: falta de investigadores, altos costos de I+D, falta de la infraestructura adecuada, ausencia de apoyos públicos para los proyectos.

Por su parte, los factores que promueven la colaboración entre industria y IES son: el prestigio de profesores calificados que se manifiestan dispuestos a ejecutar proyectos vinculados; objetivos institucionales orientados a la vinculación; así como, la infraestructura y equipamiento adecuados para la ejecución de proyectos con las empresas.

Entre los aspectos que benefician esta vinculación se destacan el incrementar fondos para financiar proyectos, aumentar la visibilidad de las capacidades institucionales para realizar proyectos innovadores, el buen manejo de la propiedad intelectual y la sensibilización de empresarios sobre los beneficios que puede reportar la colaboración con las IES (Vélez-Cuartas, 2017)

Asimismo, la interacción entre la industria y las IES generan el retorno tecnológico, reducción de costos del desarrollo y transferencia (Bell, 1993); la mejora del proceso de empleabilidad de egresados, así como, la capacitación para el personal de la empresa; la reducción de la dependencia tecnológica respecto a empresas de otros países; y mayor control sobre los activos intelectuales. El término "retorno tecnológico" se refiere a las contribuciones tanto para el gobierno u otras organizaciones públicas o privadas encargadas de financiar proyectos de $\mathrm{I}+\mathrm{D}$. Este retorno deriva de resultados como el desarrollo de nuevos productos; introducción de nuevos equipos de I+D; nuevas unidades de negocio; apertura de nuevos mercados; aumento de la exposición internacional; el aprendizaje tecnológico; mejores relaciones de la empresa con el ecosistema de innovación; y un mejor rendimiento tecnológico global.

Por su parte, las universidades se benefician de dicha interacción con la industria al obtener recursos extraordinarios, exponer a sus investigadores y estudiantes a problemas reales, fortalecer sus relaciones con el ecosistema, y aumentar su patrimonio intelectual.

La OTT se encarga de concretar estos beneficios, a partir de su estrategia de comercialización de conocimientos hacia posibles usuarios del sector privado y público (Siegel et al., 2003). De ahí la importancia de su adecuada gestión.

\section{MARCO CONCEPTUAL}

Se utiliza en esta investigación el modelo de la triple hélice como base para interrelacionar esferas interinstitucionales que integran la transferencia tecnológica y, por consiguiente, impulsan el desarrollo tecnológico y económico basado en el conocimiento. Para propósitos de nuestro estudio se concibe la Triple Hélice como la interacción tríadica entre el Estado (E), la Academia (A) y la Industria (I) Etzkowitz (2000). Para la adecuada colaboración entre estas tres organizaciones, la idea de hélices denota que se trasciende la cooperación simple para dar lugar a relaciones interactivas dentro del contexto de la transferencia tecnológica que pueden llevar al intercambio puntual de roles. La universidad incursiona en actividades empresariales con sus instalaciones de incubación; la industria educa a 
través de universidades empresariales; mientras que el gobierno se convierte en un capitalista de riesgo a partir de su inversión en $\mathrm{I}+\mathrm{D}$ e impulsa a los otros sectores a resolver cuestiones de competitividad nacional. Es a partir del modelo de triple hélice que se promueve la misión emprendedora de la universidad, siendo ésta la base fundamental entre las relaciones gobierno- industria- academia.

Esa interacción es crucial para que se incremente la transferencia tecnológica en las OTT de las instituciones de educación superior (figura $\mathbf{1}$ a). La figura $\mathbf{1 b}$ se muestra un modelo conceptual propuesto para las universidades públicas mexicanas a partir de la triple hélice, como es el caso de Necoechea et al. (2013), quienes exponen que debido a que México no es aún una economía basada en conocimiento, existen dos retos para articular su triple hélice: el primero es crear canales efectivos de TT para traducir la capacidad de investigación y capital humano en activos para crecimiento económico, competitividad e incremento del estándar de vida. El segundo es mejorar la competitividad y productividad en empresas pequeñas y medianas. Basándose en esas premisas, los autores consideran que el modelo propuesto mejorará los mecanismos existentes de TT, incrementará la innovación; e incrementará la accesibilidad de las instituciones de investigación.

\section{Figura 1}

Modelo de la triple hélice

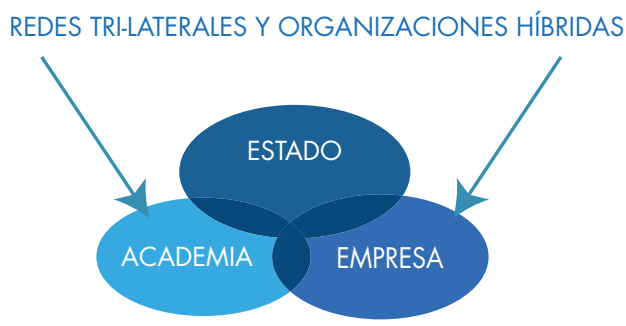

a)Fuente: Etzkowitz, H and Leydesforff, L. (2000)
Modelo conceptual TT en universidades públicas en México

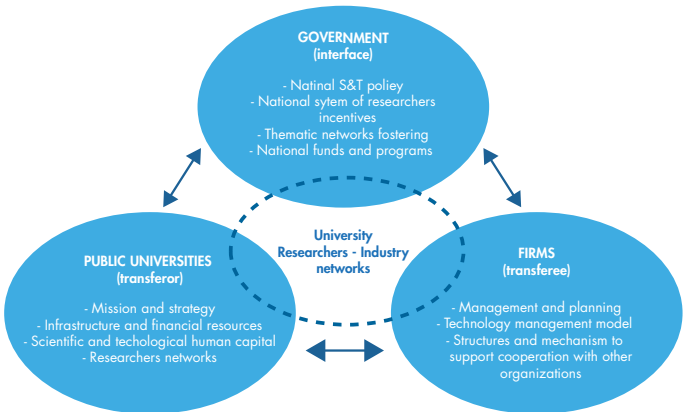

b) Fuente: Necoechea et al., (2013)

Para lograr esta articulación, es importante atender a conceptos de la teoría del capital humano, ya que pueden ayudar a los profesionales a entender el tipo de entrenamiento y habilidades que deben desarrollar en los trabajadores, así como la forma en que las decisiones directivas afectan sus intereses. Esta teoría lleva también a comprender que un mayor compromiso y liderazgo del gobierno para aumentar el nivel de inversión en educación y entrenamiento incrementará la competitividad nacional e incrementará tanto los beneficios sociales como el desempeño de las empresas.

Por lo anterior, este estudio se dedica no sólo a identificar las habilidades técnicas sino también las habilidades de gestión en el agente de transferencia tecnológica para poder identificar su perfil idóneo.

\section{METODOLOGÍA}

\section{a) Diseño de la investigación}

Se realizó una revisión sistemática de la literatura existente y relevante, en forma deductiva, y definida por las palabras clave de transferencia de tecnología; modelos y agentes de transferencia de tecnología; gestión de capital humano. En dicha revisión se analizó el estado del arte y la aplicación de dichos modelos. Esta búsqueda se realizó en 56 artículos de revistas científicas arbitradas, así como, en 38 páginas web oficiales de organismos públicos y privados. Fueron seleccionados 27 artículos científicos, 13 páginas de internet, 5 documentos oficiales, de acuerdo con el criterio de pertinencia para el objetivo de la investigación. La mayoría de los artículos utilizados son de los 
últimos 10 años, todas las páginas de internet fueron consultadas durante el mes de febrero del 2017.

Para el estudio empírico que se llevó a cabo se identificaron un total de 35 OTT de instituciones de educación superior en la República Mexicana, identificadas en la página oficial de la Red de Oficinas de Transferencia de Tecnología en México, avalada por el Conacyt y la Secretaría de Economía. El total de las OTT registradas en México en 2017 era de 131. Para objeto del presente estudio, de la población existente únicamente se seleccionaron las OTT pertenecientes o adjuntas a instituciones de educación superior, las cuales fueron 35. El cuestionario se envió a la totalidad de OTT de universidades públicas y privadas incorporadas a la red de OTT (9 fueron consultadas para efectos del pilotaje del instrumento). Se realizó una división de 5 áreas geográficas en el país (Noroeste, Norte, Occidente, Centro y Sur).

Finalmente, se identificó la información de contacto de los agentes encargados de las OTT de instituciones de educación superior. Después, se les envió por medio electrónico a los agentes de estas OTT una carta con la explicación de la investigación e invitación para contestar el cuestionario en línea. Este proceso inició desde finales de febrero y finalizó a finales de mayo de 2017. Se recibió respuesta de 34 OTT universitarias, lo cual representa una tasa de respuesta del $99 \%$, de las cuales $60 \%$ son públicas y $40 \%$ son privadas. De estas respuestas, todas se recibieron completas por lo que no se descartó ningún cuestionario. Así, los cuestionarios utilizados fueron 34, siendo 25 de universidades, 5 de institutos tecnológicos y 4 de politécnicos.

\section{b) Diseño del instrumento empírico: desarrollo y selección de variables para perfil.}

Se desarrolló un instrumento para describir las características que debiera tener el perfil del líder de la OTT. Existen diferentes modelos que analizan las competencias y habilidades gerenciales. Entre los más conocidos y considerados dentro de este artículo se encuentran los siguientes:

-El modelo de Katz (1955) que tiene tres habilidades, entre las que se encuentran las técnicas, humanas y conceptuales.

-El modelo de Reuven Bar-On (1985) sobre inteligencia social emocional que tiene dos partes, la teórica y métrica.

-El modelo de Salovey and Mayer (1990) de inteligencia emocional que contiene cuatro categorías: entendimiento, gestión, uso y percepción de emociones.

-El modelo de Goleman (1991) considera dos aspectos, el cognitivo y el de personalidad.

Derivado de un estudio de competencias se generó una clasificación de habilidades gerenciales y técnicas o profesionales. Dentro de cada una de las habilidades se agruparon las habilidades -factores- que serían las más relevantes. Dentro de las técnicas, se consideraron desde los logros académicos, conocimientos tecnológicos, dominio en áreas de negocios, procedimientos internos y normatividad, propiedad industrial, planeación y resolución de problemas, hasta la experiencia dentro de su área. Mientras que en la parte de atributos gerenciales se encuentran comunicación asertiva, negociación, clima laboral, emprendedurismo corporativo, ética, liderazgo, vinculación y creatividad.

\section{a. Obtención de datos: Muestra reducida - explicación.}

La ANUIES clasifica al Sistema de Educación Superior en los siguientes subsistemas: a) universidades públicas federales y estatales, las cuales en su gran mayoría son autónomas; b) universidades tecnológicas; c) universidades particulares, las cuales requieren del reconocimiento de validez oficial de estudios de la SEP o de los gobiernos estatales, o bien, pueden estar incorporadas a una institución educativa pública facultada para ello; d) institutos tecnológicos, los cuales son coordinados en su gran mayoría por la SEP; e) escuelas normales; f) otras instituciones públicas (ANUIES, 2000a). 


\section{a. 1. 2. Selección de la muestra.}

La selección de la muestra a considerar en el estudio se realizó, por muestreo estadístico, el cual es un procedimiento para obtener datos de una población con la finalidad de usar esta información para realizar inferencias acerca de dicha población mediante las técnicas de la estadística inferencial. (Poveda, 2017).

Para este caso en particular se consideran los siguientes factores, el tamaño de la población (N) es de 35 oficinas de transferencia tecnológica de IES a nivel nacional que están registradas ante la red nacional de OTT, se determinó considerar un nivel de confianza de $95 \%$, es decir la probabilidad de que la muestra que se ha elegido influye en los resultados obtenidos, y un margen de error del $12 \%$.

El tamaño de la muestra es de 33 oficinas de transferencia tecnológica del total de la población con un nivel de confianza del $95 \%$ y un margen de error del $5 \%$. Por consiguiente, si consideramos el factor de muestreo que resulta de dividir el tamaño de la muestra entre el tamaño de la población, se obtiene un valor del $68 \%$. Es decir; el tamaño de la muestra representa el $68 \%$ de la población y cada muestra representa 1.4 elementos de la muestra; por lo que aproximadamente es una proporción de 1 a 1.

\section{b.2. Validación del instrumento.}

Para la prueba piloto se determina considerar al resto de la población en este caso 9 oficinas de transferencia tecnológica de IES, a las cuales se enviará la encuesta para que sea validada y con la realimentación que se obtenga se realizarán los ajustes sugeridos por los expertos para que posteriormente sea aplicada a la muestra seleccionada y así obtener los datos que nos permitan realizar el análisis del perfil del agente de transferencia tecnológica en las IES de México.

Posterior a la aplicación del instrumento -encuesta- a todos los líderes representantes de las OTT de las universidades a nivel nacional, distribuida por medio electrónico, pero con un previo contacto telefónico y en algunos casos por medio de videoconferencia para acortar la distancia geográfica, espacial y relacional, se validó el instrumento mediante el cálculo del coeficiente de alfa de Cronbach con base al coeficiente de correlación de cada uno de los ítems.

Por tanto, para el instrumento que consta de 22 constructos y que agrupa las diferentes habilidades para el perfil de un agente de transferencia, se tiene un total de 64 ítems para recopilar la información que se utilizó para el cálculo del coeficiente por tanto tenemos que:

$$
\text { Cronbach's Alpha }=\mathbf{0 . 8 8 0}
$$

De acuerdo a este coeficiente podemos considerar que 0.88 se encuentra dentro del límite de 0.7 a 0.9 que indica una buena consistencia interna para esta escala.

\section{RESULTADOS}

Antes de iniciar el análisis de componentes principales- PCA (principal components analysis), se realiza un análisis previos de los datos, lo que permitió, en primera instancia, identificar que aquellos datos relacionados con preguntas de ética (variable 48,50) tienen un comportamiento atípico, ya que se tiene un valor de la media igual a 5 , que es el valor máximo que se puede obtener para cada una de las variables, esto muestra que el valor de la varianza es cero, lo que indica que la formulación de las preguntas para la obtención de estos datos tiene que reformularse y utilizar preguntas confirmatorias o cruzadas de tal manera que se pueda verificar las respuesta. Se puede argumentar que el hecho de ser preguntas directamente realizadas al comportamiento ético es posible que se haya provocado un sesgo por parte del investigador por lo que se requiere de una estrategia que permita la obtención de datos válidos. 
Tabla 2.

Varianza total explicada por los componentes.

Varianza total explicada

\begin{tabular}{c|c|c|c}
\hline \multirow{2}{*}{ Componente } & \multicolumn{3}{|c}{ Autovalores iniciales } \\
\cline { 2 - 4 } & Total & \% de la varianza & \% acumulado \\
\hline 1 & 12.625 & 20.363 & 20.363 \\
\hline 2 & 8.964 & 14.458 & 34.821 \\
\hline 3 & 5.977 & 9.64 & 44.461 \\
\hline 4 & 5.618 & 9.061 & 53.522 \\
\hline 5 & 4.542 & 7.325 & 60.847 \\
\hline 6 & 4.304 & 6.943 & 67.79 \\
\hline 7 & 3.637 & 5.867 & 73.657 \\
\hline 8 & 3.483 & 5.618 & 79.275 \\
\hline
\end{tabular}

Método de extracción: Análisis de Componentes principales.

Fuente: elaboración propia.

Por lo tanto en los resultados del análisis factorial por PCA obtenidos, por correlación se muestra en tabla 3 para seleccionar el número de componentes se consideran tres criterios, el total de la varianza explicada por los componentes y el valor de los valores propios $(>1)$ y el grado de significancia, de acuerdo con esto se consideran 12 componentes que, en conjunto, explican el $94 \%$ de la varianza total del total de las 62 variables, que tiene un valor superior a 1, que se complementa con el gráfico de sedimentación (anexo 5) de los valores propios, donde se muestra que, de acuerdo al criterio de selección (donde la gráfica tiene un "codo"), es su trayectoria.

Después de haber obtenido 342 coeficientes, a partir de esta discriminación basada en el porcentaje acumulado, se consideraron únicamente 42 coeficientes, los cuales engloban el $94 \%$. A partir de ello se obtuvieron 17 coeficiente dentro del primer factor, 12 en el segundo, 7 en el tercero y 5 en el cuarto.

De tal forma que la nueva clasificación por constructos es la siguiente:

\section{Tabla 3.}

Tabla con constructos de versión final.

\begin{tabular}{l|l}
\multicolumn{1}{c|}{ Dimensión } & \multicolumn{1}{c}{ Sub-dimensión } \\
\hline Negociación, vinculación y organización. & $\begin{array}{l}\text { Habilidades de negociación } \\
\text { Habilidades de vinculación } \\
\text { Habilidades de organización }\end{array}$ \\
\hline Experiencia y conocimientos en: & $\begin{array}{l}\text { Proyectos } \\
\text { Planeación } \\
\text { Control de riesgos }\end{array}$ \\
\hline Habilidades de comunicación enfocadas a la promoción de TT. \\
\hline Formación en gestión de proyectos de TT.
\end{tabular}

Fuente: Elaboración propia basada en los resultados obtenidos. 
A través del análisis, se agruparon las habilidades analizadas de acuerdo con sus similitudes, se descartaron las que tienen una baja relación por su coeficiente de correlación, lo que permite reducir el número de variables dentro del instrumento y agilizar las respuestas para los encuestados en próximas investigaciones.

\section{ANÁLISIS DE RESULTADOS}

Respecto al análisis de los representantes de las OTT en México se encuentran los siguientes resultados. La población que labora en esta industria está conformada de un $50 \%$ de hombres y $50 \%$ de mujeres. Es un ámbito donde sí tienen cabida las mujeres tanto para adquirir experiencia como para tomar decisiones relacionadas con la ciencia en México.

El nivel académico es mayoritariamente de educación superior a nivel de posgrado, ya que $61.4 \%$ estudio maestría; $31.77 \%$ cuenta con doctorado y universidad $7.69 \%$. Afirmaron en $61.54 \%$ que su formación académica les permite comprender los desarrollos tecnológicos que transfieren, mientras que sólo 10\% estuvieron en desacuerdo con esta afirmación. Entre los tipos de formaciones se encuentran gestión de la innovación tecnológica 31 \%, transferencia tecnológica $9 \%$, propiedad intelectual $3 \%$ y, $56 \%$, administración de proyectos, mercadotecnia, emprendedurismo, áreas fundamentales para el desarrollo de transferencia tecnológica.

Entre los cargos que tienen los representantes, $39 \%$ son directores, $31 \%$ jefes, 27 por ciento coordinadores y $4 \%$ subdirectores. El porcentaje de participación por región es de 35 \% para la región centro que incluye a la ciudad de México, Estado de México y Puebla que tiene la mayor concentración de instituciones, seguido de la región noroeste con $27 \%$, región occidente $15 \%$, región noreste $11 \%$ y la región sur con un $12 \%$. Concerniente a su edad, un poco más de la mitad (53.9\%) tiene más de 45 años de edad; un $30.77 \%$ se encuentra entre 30 y 45 años de edad y sólo el $15.38 \%$ tiene menos de 30 años de edad. El 42 \% ha colaborado en transferencia tecnológica entre 5 y 10 años; es decir, la mitad de los representantes inició su experiencia en esta área entre los 35 y 40 años de edad. Es decir, la mayor parte de los expertos que dirigen una OTT tuvo experiencia laboral previa y se puede inferir que el género femenino tiene presencia en el gremio. Los participantes afirmaron tener en su mayoría experiencia en planeación (92.3\%), en comercialización (73.07 \%), recursos humanos (65.39), mercadotecnia (61.54\%), legal (42.31 \%), producción (38.46 \%).

Por otra parte, de las siete habilidades técnicas que se consideraron puntualmente como: propiedad intelectual, emprendimiento, planeación de negocios, mercadotecnia, manejo de software de office administrativo, segundo idioma y programas de análisis, son las primeras cuatro en que los representantes afirman dominarlas totalmente o con un alto dominio. Mientras que las de segundo idioma y programas de análisis, tuvieron mayor dispersión en sus respuestas. Respecto al segundo idioma, al $60 \%$ que lo domina totalmente y al $15 \%$ en programas de análisis.

Se observó que, para los agentes de transferencia tecnológica, tanto la negociación como la comunicación son elementos clave no sólo para desempeñar las actividades de transferencia sino para el éxito de las mismas. La parte de comunicación tiene un área de oportunidad, ya que las ideas propuestas a los clientes potenciales son menos aceptadas que las propuestas a los clientes actuales y colaboradores.

\section{CONCLUSIONES Y TRABAJOS FUTUROS}

Se puede concluir que, particularmente para el caso de México, ser agente de transferencia tecnológica implica un camino arduo, compuesto tanto de experiencia y conocimiento en áreas específicas sobre transferencia tecnológica como de habilidades gerenciales para que se alcance el objetivo propuesto. Después de análisis de las variables se identificó que, si bien las habilidades técnicas son fundamentales para la planeación y ejecución de la transferencia tecnológica, las habilidades gerenciales y particularmente las interpersonales son las que predominan como habilidades para obtener una exitosa transferencia tecnológica; dado que son las que suavizan las asperezas, las que integran y vinculan a los integrantes y las que fortalecen los lazos sobre todo en momentos de crisis, por ejemplo para la elaboración de los planes de contingencia.

El análisis PCA permitió identificar que los datos relacionados con preguntas de ética se caracterizan por un comportamiento anormal, por consiguiente, el instrumento debe reformularse para recabar datos que puedan verificar el comportamiento ético para eliminar el sesgo por parte del investigador por medio de estrategias que inciden en establecer la objetividad y la equidad como criterios explícitos.

Las habilidades que no se consideraron explícitamente en este estudio, pero que fueron mencionadas por los agentes de transferencia tecnológica en las entrevistas aplicadas son: pasión, liderazgo, estar abierto al cambio, capacidad 
de aprendizaje y disciplina. Éstas se mencionaron como fundamentales para que una transferencia tecnológica sea exitosa. Debido a que una negociación requiere tanto de automotivación como de motivar al equipo; de ser empático y saber escuchar para entender las necesidades de los participantes; ética y confianza para que clientes internos y externos compartan sus resultados tecnológicos, tomen la decisión de invertir y contemplen el proceso con visión a largo plazo. Los encuestados afirman que la visión de negocio es idónea para identificar oportunidades de transferencia tecnológica. Por su parte, la creatividad y proactividad son básicas para solucionar problemas o bien para proponer planes de contingencia, al igual que la capacidad de negociación y solución de conflictos interpersonales.

Por otra parte, se mencionó que, existen diversas formas de propiciar la vinculación, tales como: relaciones de amigos, congresos y conferencias. Al mismo tiempo, se constató que aún existen diversas barreras para transferir tecnología de las universidades, tales como: las políticas universitarias poco flexibles, la falta de reglamentación interna en materia de propiedad intelectual; burocracia de la estructura organizacional para la aprobación de proyectos de transferencia tecnológica; así como, de la aplicación de los ingresos generados.

Entre las principales aportaciones de este estudio se encuentra el hacer énfasis en que las habilidades gerenciales son indispensables para el desarrollo del trabajo de un agente tecnológico y que esas habilidades no forman parte de la currícula de estudios formales. Asimismo, se considera que este estudio está entre los pioneros a nivel nacional sobre el tema, y ofrece una base para futuras investigaciones tanto teóricas como prácticas para profundizar en el ámbito del capital humano para las OTT. 


\section{REFERENCIAS}

Algieri, B., Aquino, A., \& Succurro, M. (2013). Technology transfer offices and academic spin-off creation: the case of Italy. The Journal of Technology Transfer, 38(4), 382-400.

ANUIES (2000a). La ANUIES en la línea del tiempo. 50 años de historia. ANUIES, México.

Bell, E. R. (1993). Some current issues in technology transfer and academic-industrial relations: a review: Practitioners' forum. Technology Analysis \& Strategic Management, 5(3), 307-322.

Beltrones, A. V. G., Miranda, G. A. V., Ontiveros, M. D. R., Ortíz, K. R., \& Cázares, E. A. (2017). La Vinculación en las Universidades Públicas Versus las Universidades Privadas en México. Retos y Desafíos en el Siglo XXI. Revista Jurídica del Departamento de Derecho, 9(17 jul-dic), 47-60.

Beraza-Garmendia, J. M., \& Rodríguez-Castellanos, A. (2015). Characteristics and effectiveness of university spinoff support programmes. Academia Revista Latinoamericana de Administración, 28(1), 14-44.Bozeman, B. (2000). Technology transfer and public policy: a review of research and theory. Research policy, 29(4-5), 627-655.

Cabrero, E., Cárdenas, S., Arellano, D., \& Ramírez, E. (2011). La vinculación entre la universidad y la industria en México: Una revisión a los hallazgos de la Encuesta Nacional de Vinculación. Perfiles educativos, 33(SPE), 187199.

Casas, R. (1999). El gobierno: hacia un nuevo paradigma de política para la vinculación. R. Casas y M. Luna (Coords.), Gobierno, academia y empresa en México: Hacia una nueva configuración de relaciones, 77-118.

Corsi, C., \& Prencipe, A. (2016). Improving innovation in university spin-offs: the fostering role of university and region. Journal of technology management \&' innovation, 11(2), 13-21.

Cusi, M. L. A. (2021). Identificación de las capacidades tecnológicas de la Fundación Universitaria de Ciencias de la Salud, Colombia. Revista Repertorio de Medicina y Cirugía, 30(1), 29-42.

Estreicher, S., \& Yost, K. A. (2015). University IP: The University as Coordinator of the Team Production Process. Ind. LJ, 91, 1081.

Etzkowitz, H., \& Leydesdorff, L. (2000). The dynamics of innovation: from National Systems and "Mode 2" to a Triple Helix of university-industry-government relations. Research policy, 29(2), 109-123.

Fard, M. S., Cheong, K. C., \& Yap, S. F. (2014). Reopening the debate on globalisation and economic growth through technology transfer. Malaysian Journal of Economic Studies, 51(2), 231

Franks, R. P., \& Bory, C. T. (2017). Strategies for developing intermediary organizations: Considerations for practice. Families in Society, 98(1), 27-34.

Feria P, V., \& Hidalgo N, A. (2011). Towards a Transfer Model of Scientific and Technological Knowledge: the Case of Mexico (pp. 1-19): International Association for Management of Technology.

García García, A. J. (2019). Las junior empresas como complemento a la formación de los estudiantes universitarios y propuesta de un modelo de integración.

González, J.D. (2015), "Diagnóstico del capital humano de las OTC en México: propuesta para su profesionalización”, Tesis de Maestría, Centro de Ciencias Económicas, Administrativas y Sociales, Instituto Politécnico Nacional, México, D.F.

Harman, G. (2010). Australian university research commercialisation: perceptions of technology transfer specialists and science and technology academics. Journal of Higher Education Policy and Management, 32(1), 69-83 doi: $10.1080 / 13600800903440568$.

Harman, G., \& Stone, C. (2006). Australian university technology transfer managers: Backgrounds, work roles, specialist skills and perceptions. Journal of Higher Education Policy and Management, 28(3), 213-230. doi:10.1080/13600800600979959.

Hrehová, D., \& Jencová, A. (2014). Key attributes of the personality of the entrepreneur in the perception of students of technical specialization. International Multidisciplinary Scientific Conference On Social Sciences \& Arts SGEM, 475-482. 
Junior, M. V., Wagner, C. L., Vanalle, R. M., \& Jagoda, K. (2014). Effective management of international technology transfer projects: Insights from the Brazilian textile. Journal of Manufacturing Technology Management, Vol. 25 Issue: 1, pp.69-99, doi: 10.1108/JMTM-08-2011-0079.

Leiponen, A. (2005). Skills and innovation. International Journal Of Industrial Organization, 23(5/6), 303-323. doi:10.1016/j.ijindorg.2005.03.005.

Lichtenthaler, U., \& Ernst, H. (2009). The Role of Champions in the External Commercialization of

Makarov, I., \& Plantin, G. (2015). Rewarding Trading Skills without Inducing Gambling. Journal Of Finance, 70(3), 925-962. doi:10.1111/jofi.12257.

Marion, T. J., Dunlap, D. R., \& Friar, J. H. (2012). The university entrepreneur: a census and survey of attributes and outcomes. REDD Management, 42(5), 401-419. doi:10.1111/j.1467-9310.2012.00691.x.

Mom, T. J., Oshri, I., \& Volberda, H. W. (2012). The skills base of technology transfer professionals. Technology Analysis ES Strategic Management, 24(9), 871-891. doi:10.1080/09537325.2012.718663.

Necoechea, H., Pineda-Domínguez, D., \& Soto-Flores, R. (2013). A conceptual model of technology transfer for public universities in Mexico. Journal of technology management \& innovation, 8(4), 24-35.

OCDE (2009). Science, Technology and Industry Scoreboard 2009, París, OCDE.

O'Kane, C., Mangematin, V., Geoghegan, W., \& Fitzgerald, C. (2015). University technology transfer offices: The search for identity to build legitimacy. Research Policy, 44(2), 42 1-437. doi:10.1016/j.respol.2014.08.003.

Olaya, E. S., Berbegal-Mirabent, J., \& Germán, D. O. (2014). Desempeño de las oficinas de transferencia universitarias como intermediarias para la potencialización del mercado de conocimiento. Intangible Capital, 10(1), 155-188. doi: $10.3926 /$ ic. 497 .

Ragsdell, G., Rathi, D., Given, L. M., \& Forcier, E. (2016). Knowledge needs in the non-profit sector: an evidence-based model of organizational practices. Journal of Knowledge Management, 20(1), 23-48.

Red de oficinas de Transferencia de Tecnología en México, Conacyt - Secretaría de Economía, www.redott.com.mx consultada en el mes de Febrero del 2017

Riviezzo, A. y M.R. Napolitano (2010), "Italian Universities and the Third Mission. A longitudinal analysis of organizational and educational evolution towards the "entrepreneurial university", Industry and Higher Education, vol. 24, núm. 3, pp. 227-236.

Rothaermel, F.T., Agung, S.D. \& Jiang, L. (2007) University Entrepreneurship: a taxonomy of the literature, Industrial and Corporate Change, 16(4), 691-791. http://dx.doi.org/10.1093/icc/dtmo23

Sarin, S., \& McDermott, C. (2003). The Effect of Team Leader Characteristics on Learning, Knowledge Application, and Performance of Cross-Functional New Product Development Teams. Decision Sciences, 34(4), 707-739. doi: $10.1111 / \mathrm{j} .1540-5414.2003 .02350 . x$.

Siegel, D.S., D.A. Waldman and A.N. Link (2003), 'Assessing the impact of organizational practices on the productivity of university technology transfer offices: an exploratory study,' Research Policy, 32(1), 27-48.

Solleiro, J.L. (2009), Operación de oficinas de transferencia de tecnología en Lizardi, V. y Vázquez, L. Oficinas de transferencia de tecnología. Fundamentos para su formación y operación en México, Asociación de Directivos de la Investigación Aplicada y el Desarrollo Tecnológico (ADIAT), México, D.F. 222-242

Spithoven, A., \& Knockaert, M. (2012). Technology intermediaries in low tech sectors: The case of collective research centres in Belgium. Innovation: Management, Policy \& Practice, 14(3), 375-387.

Sterckx, S. (2011). Patenting and Licensing of University Research: Promoting Innovation or Undermining Academic Values? Science E Engineering Ethics, 17(1), 45-64. doi:10.1007/s1 1948-009-9168-8.

Suvinen, N., Konttinen, J., \& Nieminen, M. (2010). How Necessary are Intermediary Organizations in the Commercialization of Research? European Planning Studies, 18(9), 1365-1389. doi:10.1080/09654313.2010.492 584 .

Trijillo, N. L., \& Everett, B. A. (2010). Technology Transfer and Intellectual Property Issues. New York: Nova Science Publishers, Inc. 
Ustundag, A., Ugurlu, S., \& Kilinc, M. S. (2011). Evaluating the performance of technology transfer offices. Journal of Enterprise Information Management, Vol. 24 Issue: 4, pp.322-337, doi: 10.1108/17410391111148576.

Vélez-Cuartas, G. J., Uribe-Tirado, A., Robledo-Velásquez, J., Restrepo, D., Correa, M. C., Pallares, C., ... \& Castro, A. M. (2017). Indicadores de vinculación con el entorno para Unidades de Gestión de Investigación.(Universidad de Antioquia-SIU. Estudio Piloto 2004-2016).

Villani, E., Grimaldi, R. \& Rasmussen E. (2015). How intermediary organizations facilitate university-industry technology transfer: A proximity approach. Technological Forecasting \& Social Change.

Vorley T. and Nelles J. (2009). Building Entrepreneurial Architectures: A Conceptual Interpretation of the Third Mission. Policy Futures in Education. Vol 7, Issue 3, pp. 284- 296. First published date: January-01-2009 10.2304/ pfie.2009.7.3.284.

Villani, E., Rasmussen, E., \& Grimaldi, R. (2015). How Intermediary Organizations Facilitate Technology Transfer: A Proximity Approach. Academy Of Management Annual Meeting Proceedings, 1. doi:10.5465/ AMBPP.2015.13440abstract.

Vutsova, A., \& Ignatova, O. o. (2014). The role of public-private partnership for effective technology transfer. Applied Technologies \& Innovations, 10(3), 83-90.

Wolfe, A. K., et al.(2013). Insiders' views of the valley of death: Behavioral and institutional perspectives. BioScience, 64(2), 138-144 doi:10.1093/biosci/bit015. 


\section{Figura 2}

Ubicación geográfica de las Oficinas de Transferencia Tecnológica en Universidades Mexicanas.

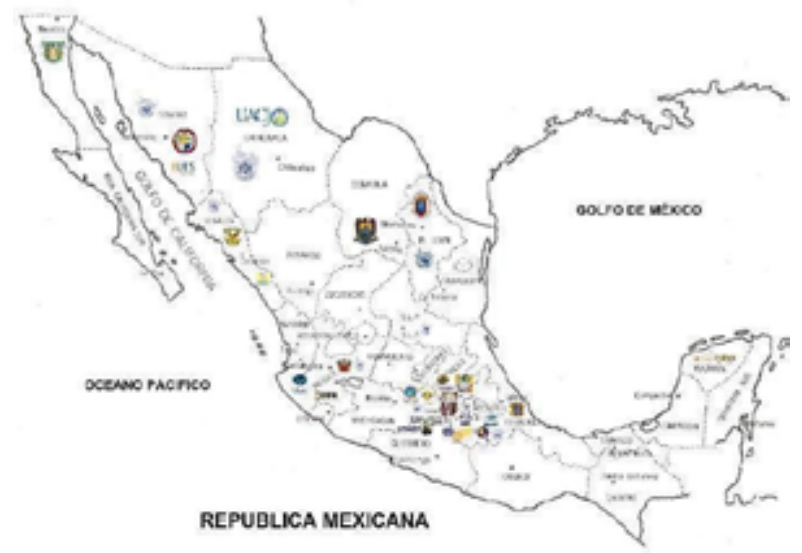

Fuente: Elaboración propia basado en la muestra específica seleccionada de IES.

\section{Anexo 1}

Matriz de componentes

Matriz de componentesa

\begin{tabular}{|c|c|c|c|c|c|c|c|c|c|c|c|c|c|c|}
\hline & \multicolumn{14}{|c|}{ Componente } \\
\hline & 1 & 2 & 3 & 4 & 5 & 6 & 7 & 8 & 9 & 10 & 11 & 12 & 13 & 14 \\
\hline V3 & & & & & & & & .590 & & & & & & \\
\hline V4 & & & .658 & & & & & & & & & & & \\
\hline V5 & & & .556 & & & & -.630 & & & & & & & \\
\hline V6 & & & \begin{tabular}{|l|l|} 
\\
\end{tabular} & & & & & & & & & & & \\
\hline V7 & & & & .702 & & & & & & & & & & \\
\hline V8 & & & & .623 & & & & & & & & & & \\
\hline V9 & & & & & & & & & .634 & & .508 & & & \\
\hline V11 & & & & .708 & & & & & & & & & & \\
\hline V12 & & & & & & & & & & -.547 & & & & \\
\hline V13 & & & .723 & & & & & & & & & & & \\
\hline V14 & & & & & & & & .517 & & & & & & \\
\hline V15 & & .847 & & & & & & & & & & & & \\
\hline V16 & & .792 & & & & & & & & & & & & \\
\hline V17 & .509 & .739 & & & & & & & & & & & & \\
\hline V18 & & .871 & & & & & & & & & & & & \\
\hline V20 & & .674 & & & & & & & & & & & & \\
\hline V21 & & .747 & & & & & & & & & & & & \\
\hline V22 & & .888 & & & & & & & & & & & & \\
\hline V23 & .598 & & & & & & & & & & & & & \\
\hline V24 & & .823 & & & & & & & & & & & & \\
\hline V25 & & & & .544 & & & -.503 & & & & & & & \\
\hline V26 & & & & & & & & & .513 & & & & & \\
\hline
\end{tabular}




\begin{tabular}{|c|c|c|c|c|c|c|c|c|c|c|c|}
\hline V27 & & & & & .904 & & & & & & \\
\hline V28 & & & & .708 & & & & & & & \\
\hline V29 & & & & & & -.683 & & & & & \\
\hline V30 & & .641 & & & & & & & & & \\
\hline V31 & .720 & & & & & & & & & & \\
\hline V32 & .663 & & & & & & & & & & \\
\hline V33 & .708 & & & & & & & & & & \\
\hline V34 & .803 & & & & & & & & & & \\
\hline V35 & .634 & & & & & & & & & & \\
\hline V36 & .748 & & & & & & & & & & \\
\hline V37 & .643 & & & .601 & & & & & & & \\
\hline V38 & & .733 & & & & & & & & & \\
\hline V39 & .635 & & & & & .678 & & & & & \\
\hline V40 & .594 & & & & & & & & & & \\
\hline V41 & & & & & -.519 & & & & & & \\
\hline V42 & & & & & & & & & & & .529 \\
\hline V43 & .754 & & & & & & & & & & \\
\hline V44 & .582 & & & & & & & & & & \\
\hline V45 & .576 & & & & & & & & & & \\
\hline V46 & .639 & & & & & & & -.569 & & & \\
\hline V47 & & & & & & & & & & -.516 & \\
\hline V48 & & & & & & & .509 & & & & \\
\hline V49 & & & & & & & & & .526 & & \\
\hline V51 & & & .518 & & & & & & & & \\
\hline V53 & & & & & & .601 & & & & & \\
\hline V54 & & & .586 & & & & & & & & \\
\hline V55 & .526 & & & & & & & & & & \\
\hline V56 & .528 & & & & & & & & & & \\
\hline V58 & .519 & & & & & & & & & & \\
\hline V59 & & .557 & & & & & & & & & \\
\hline V61 & & & .505 & & & & & & & & \\
\hline V62 & .685 & & & & & & & & & & \\
\hline V63 & .791 & & & & & & & & & & \\
\hline V64 & .724 & & & & & & & & & & \\
\hline V65 & .575 & & -.561 & & & & & & & & \\
\hline V66 & .786 & & & & & & & & & & \\
\hline
\end{tabular}

a. 14 componentes extraídos

Fuente: Elaboración propia de los autores. 